\title{
Carnets
}

Revue électronique d'études françaises de l'APEF

Première Série - 1 Numéro Spécial | 2009

Cultures littéraires : nouvelles performances \& développement

\section{Pluralisme axiologique ou cohérence culturelle: l'enseignement du champ de la littérature interculturelle}

\section{Margarita Alfaro Amieiro}

\section{(2) OpenEdition}

\section{Journals}

Édition électronique

URL : http://journals.openedition.org/carnets/4176

DOI : $10.4000 /$ carnets. 4176

ISSN : 1646-7698

Éditeur

APEF

Édition imprimée

Date de publication : 1 juin 2009

Pagination : 351-363

\section{Référence électronique}

Margarita Alfaro Amieiro, «Pluralisme axiologique ou cohérence culturelle: l'enseignement du champ de la littérature interculturelle », Carnets [En ligne], Première Série - 1 Numéro Spécial | 2009, mis en ligne le 16 juin 2018, consulté le 19 avril 2019. URL : http://journals.openedition.org/carnets/4176 ; DOI : $10.4000 /$ carnets. 4176

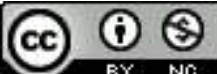

Carnets est mis à disposition selon les termes de la licence Creative Commons - Atribution - Pas d'utilisation commerciale 4.0 International. 


\title{
PLURALISME AXIOLOGIQUE OU COHÉRENCE CULTURELLE: l'enseignement du champ de la littérature interculturelle
}

MARGARITA ALFARO AMIEIRO

Université Autonome de Madrid margarita.alfaro@uam.es

\begin{abstract}
Résumé
L'enseignement de la littérature nous situe au cœur de certaines contradictions mises en valeur par les réformes des études universitaires. D'une part, la littérature est conçue en tant que transmission de plusieurs ensembles historiques dont l'un des plus importants est une approche diachronique de la culture. De l'autre, s'impose la tendance à supprimer la vision historique et à considérer la littérature comme la projection d'un système cohérent lié à l'actualité culturelle où certains contenus disparaissent en faveur de la simplification. Or, l'enseignement de la littérature peut être conçu comme un ensemble complexe articulé autour d'interférences diverses où l'étudiant apprend à se situer, aux plans conceptuel et figuratif, au moyen du raisonnement analogique et à ressentir la littérature, en partant de la polarité littérature nationale - littérature interculturelle, comme une expérience esthétique marquée par l'hybridation.
\end{abstract}

\begin{abstract}
The teaching of literature, place us in the centre of some contradictions that characterise the reforms of the actual university studies. From one side literature is understood as the transmission of multiple historical wholes where the most significant is a diachronic cultural approach. On the other, prevails in a progressive way the actual trend of leaving out the historic vision and to consider literature as a projection of a coherent system linked to the cultural present where some contents disappear for simplification. Literature teaching can be understood as a complex group formed by diverse interferences where the student can learn to place himself, whether in a conceptual or in a figurative level, in the middle of the analogical reasoning and to feel literature, starting from the polarity national literature - multicultural literature, as an esthetical experience marked by the hybridisation.
\end{abstract}

Mots-clés: Enseignement, Littérature, Interculturalité, Hybridation

Keywords: Literature teaching, Multicultural literature, Hybridization 
Ce grand monde, que les uns multiplient encore comme especes soubs genre, c'est le miroüer où il nous faut regarder pour nous connoistre de bon biais. Somme, je veux que ce soit le livre de mon escholier

Michel de Montaigne, 1969, Livre I : 205

Une Europe de polyglottes n'est pas une Europe de personnes qui parlent couramment beaucoup de langues, mais, dans la meilleure des hypothèses, de personnes qui peuvent se rencontrer en parlant chacune sa propre langue et en comprenant celle de l'autre, mais qui, ne sachant pourtant pas parler celle-ci de façon courante, en la comprenant, même péniblement, comprendraient le "génie », l'univers culturel que chacun exprime en parlant la langue de ses ancêtres et de sa tradition.

Umberto Eco

\section{Présentation $^{1}$}

L'enseignement et la diffusion des Humanités nous situent au cœur de certaines interrogations, et même de quelques contradictions, mises en valeur par les réformes de l'Enseignement Supérieur en Europe. Le rôle donné historiquement à l'Université depuis la Renaissance (la transmission des Savoirs), se transforme en faveur des exigences du monde globalisé. En effet, les textes officiels diffusés annuellement par L'UNESCO-CEPES (Centre Européen pour l'Enseignement Supérieur) indiquent que :

L'enseignement est à la fois un incubateur pour le changement externe et un innovateur pour la réforme interne. L'enseignement supérieur au Vingt-et-unième siècle connaît des défis et des changements sans précédent dans son histoire concernant la qualité des programmes d'enseignement, la pédagogie, les structures organisationnelles, le financement, les enseignants, les politiques d'inscription et les offres de programmes d'étude. Un élément important des discussions sur l'enseignement supérieur concerne également son rôle para rapport à d'autres niveaux d'enseignement (UNESCO, 2005 : Éditorial).

$\mathrm{Au}$ sein de ce cadre large de l'Enseignement Supérieur en Europe, les études littéraires, liées à l'enseignement des langues étrangères, évoluent très clairement dans le panorama de l'Université, et en particulier de l'Université espagnole, vers une nouvelle

\footnotetext{
${ }^{1}$ Ce travail s'inscrit au cadre des objectifs du projet de recherche CCG08-UAM-HUM-4429 (Université Autonome de Madrid et Communauté de Madrid), consacré à l'étude de la littérature interculturelle en Europe.
} 
approche axée notamment sur des pratiques communicatives et relationnelles de l'enseignement-apprentissage de la littérature en rapport avec une langue étrangère. Force est de constater que tous les objectifs disciplinaires et méthodologiques des plans d'études précédents sont mis en jeu et qu'un horizon d'avenir s'ouvre face aux enjeux de notre temps.

\title{
La littérature nous concerne-t-elle encore? Quel sens peut-t-elle avoir pour nous?
}

\begin{abstract}
Or, cette radicale mise en cause de la littérature nous remet, nous qui prétendons l'enseigner ou la pratiquer, radicalement en cause à notre tour. Dans nos fins comme dans no moyens, dans le sens que nous attribuons aux études littéraires comme dans les méthodes que nous utilisons.
\end{abstract}

Doubrovsky, $1981: 8$

Notre réflexion (fruit de notre expérience professionnelle depuis 1990 en tant que professeur de littérature française dans un département consacré jusqu'à présent aux études philologiques traditionnelles ${ }^{2}$ ) portera sur un cas de figure singulier. C'est-à-dire, la reformulation de l'enseignement de la discipline obligatoire de littérature française (articulée actuellement autour de 6 matières en suivant le critère d'évolution chronologique) et les matières, en option, des littératures francophones, jusqu'à présent divisées en trois domaines géographiques et culturels différenciées: la littérature franco-canadienne, la littérature africaine et la littérature francophone en Europe (la Belgique et la Suisse).

A partir de la prochaine année académique l'arbre des filières des études philologiques instaurés à L'Université Autonome de Madrid, depuis les années 90, doit se réorganiser et les différentes licences (Philologie Française, Philologie Anglaise, Linguistique, Théorie Littéraire et Littérature Comparée) vont être remplacées par une Licence de Langues modernes, culture et communication, qui aura pour but d'englober, au moyen d'une articulation modulaire les études de langues modernes (espagnol, français, anglais), littérature, linguistique, didactique des L.E., théorie littéraire et littérature générale et comparée.

Donc, actuellement, lors de l'élaboration de nouveaux cursus, l'enseignement de la littérature se trouve confronté à une double réalité épistémologique. D’une part, la tradition

\footnotetext{
${ }^{2}$ La section de Philologie Française de I'Université Autonome de Madrid naît en 1989. Actuellement le Département de Philologie Française est constitué par une vingtaine de professeurs qui assurent l'enseignement de la langue, la linguistique diachronique et synchronique, la littérature, la civilisation, la didactique et la traduction des Licences de Philologie Française, Traduction et Interprétation (où le français est langue $B$ et langue $C$ ), Tourisme et français langue $B$ pour les filières des Humanités.
} 
littéraire, conçue en tant que transmission de plusieurs ensembles historiques où le plus significatif est une approche diachronique de la culture. De l'autre, surgit la tendance très forte à supprimer la vision historique et à considérer la littérature comme la projection d'un système cohérent lié à l'actualité culturelle où certains contenus se rendent invisibles et deviennent secondaires en faveur de la simplification. À cette double perspective s'ajoutent aussi de nouveaux paradigmes en termes de références théoriques, modèles d'apprentissage et outils didactiques qui méritent d'être considérés.

À cet égard, et pour établir un parallélisme avec ce qui s'est passé en France depuis quelques années, Jean-Pierre Goldenstein considère que la littérature est devenue un paradigme dialectique qui n'échappe pas aux métamorphoses de la société. II envisageait, il y a une douzaine d'années ${ }^{3}$, un renouvellement de l'activité littéraire dans un article intitulée «Savoir-lire littéraires et enseignement universitaire actuel de la littérature ». D’abord il décrit les deux grandes tendances vers lesquelles peut s'orienter la discipline de Littérature Française. Soit vers un apprentissage chronologique, donc de nature encyclopédique, soit vers une approche technique (la connaissance de certaines stratégies analytiques) en rapport avec la critique littéraire. Goldenstein soulignait déjà en 1996 l'importance de ce qu'il appelle le «contrat pédagogique» :

Un enseignement universitaire de la littérature française se doit assurer aujourd'hui un certain nombre de tâches de première urgence. La consolidation des savoirs de base ainsi que l'acquisition des principaux outils intellectuels et matériels propres à favoriser des études de Lettres, explicitement structurées à l'intérieur d'un parcours contractuel, constituent tout d'abord un objectif prioritaire. La notion de « contrat pédagogique " est ici capitale. L'étudiant doit impérativement savoir ce qu'on attend de lui, les objectifs à atteindre et les moyens à mettre en œuvre pour les atteindre.

De ce point de vue, je plaiderai pour une appropriation active et dirigée de faits littéraires systématiquement problématisés et relativisés acquise à partir de théories savantes de référence qui permettent une mainmise effective des sujets apprenants sur l'objet de leur apprentissage. La formation académique doit prioritairement faire appel aux différents systèmes critiques et théoriques aptes à permettre aux étudiants de dominer l'objet d'étude de la façon la plus précise possible de telle sorte que les savoirs acquis à partir de textes donnés soient transférables à d'autres textes et puissent favoriser une démarche personnelle concernant d'autres problématiques (Goldenstein, $1998:$ 47-48).

\footnotetext{
${ }^{3} \mathrm{~J}$-P- Godenstein a participé au Colloque Reflexiones sobre la enseñanza de la Literatura Francesa organisé par le Département de Philologie Française de L'Université Autonome de Madrid du 26 au 29 février 1996 où se sont presentées les nouvelles propositions.
} 
Nous pourrions alors considérer, de la perspective des Humanités, que la connaissance de la littérature est liée à une tradition disciplinaire qui nous renvoie à l'établissement de la notion de canon et dont le principal objectif est la perpétuation du patrimoine littéraire. De cette constatation, deux mots nous intéressent, tradition et canon, parce qu'ils nous offrent deux domaines conceptuels essentiels qui aideront à marquer notre orientation. Le mot tradition, du latin tradere, met en valeur la dimension historique des modèles, normes et formes d'une réalité identitaire donnée. Et le mot canon (associé à la littérature) est accepté en France vers la moitié du XIX siècle et il établit un inventaire d'auteurs et de textes sélectionnés, adressé aux élites et à des fins très concrètes, notamment moralisatrices, idéologiques et esthétiques (Fernández, 2008). En revanche, aujourd'hui, la formation littéraire comme transmission d'un corpus présente des changements significatifs :

Cette fonction s'est transformée progressivement depuis le début du XXe siècle, lorsque l'école est devenue un lieu de démocratisation et de modernisation des pratiques culturelles : la littérature a gardé ses valeurs, mais celles-ci ont été mises désormais au service de l'idéal démocratique. Lire et connaître les grands auteurs est considéré comme un des moyens les plus sûrs de devenir un citoyen complet en développant à la fois une culture et une exigence éthique et esthétique. II s'agit d'éduquer à une hiérarchie des valeurs et des goûts en s'appuyant sur un corpus investi de toutes les vertus et en subordonnant la lecture aux contraintes supposées de la littérature » (Dufays, $2006: 7-8$ ).

Ainsi, au seuil du XXle siècle, la notion de "valeur littéraire » évolue et l'idéal de construction identitaire est mis en question parce que «la pluralité des traditions nationales et régionales est l'une des raisons pour lesquelles l'identité européenne manque de cohérence» (Todorov, 2005). Nous constatons, donc, que depuis quelques décennies, le corpus littéraire classique est devenu objet de contestation, à la fois que d'autres pratiques culturelles s'imposent au sein de la société. Notons au passage le succès des études culturelles, sociologiques et anthropologiques qui apparemment rendent la création littéraire, au sens traditionnel, moins pertinente en faveur des sciences sociales. Toutefois la polysémie du mot culture exprime «la vulgarisation de l'anthropologie culturelle, vulgarisation qui ne va pas toujours sans contresens ou sans simplification excessive » (Cuche, 2004 : 96).

A présent, face à l'élaboration des plans d'études auxquels nous sommes confrontés, plusieurs positions se font contrepoids. D'un côté, la littérature devient une notion en rapport avec le passé et donc élitiste. De l'autre, l'université est obligée de refaire ses méthodes d'enseignement-apprentissage. En plus, la participation des étudiants dans les 
établissements de l'enseignement supérieur de même que les enquêtes de l'UNESCO à propos des besoins des étudiants soulignent : «qu'une bonne formation universitaire devrait inclure une composante personnelle et professionnelle qui permette à l'étudiant d'évoluer socialement » (UNESCO, 2005).

Or, il convient d'ajouter l'importance du rôle joué par le professeur de littérature au cadre de la transmission du savoir. Serge Doubrovsky, dans une conférence prononcée à Cerisy en 1969 ayant pour titre «Le point de vue du professeur », lors du colloque intitulé L'enseignement de la littérature, affirme :

\begin{abstract}
Dans son rapport à son objet, la perception esthétique n'est qu'un cas particulier de la perception naturelle. Si le type de jouissance particulier, qui confère à un ensemble verbal son titre de "littérature » (par opposition à des expériences non-littéraires du langage) n'est pas, d'une certaine façon donné au départ, on ne le trouvera jamais à l'arrivée. Le relativisme culturel ne supprime nullement ce phénomène : simplement, il le déplace le long de l'axe des cultures. (...) La parole publique du discours professoral perd tout sens, si elle n'est reprise, et peut-être rejetée, par le silence d'une relecture intime (Doubrovsky, 1981 : 15-16).
\end{abstract}

En conséquence, les nouvelles dispositions institutionnelles (et sociales sans doute) se penchent du côté de l'établissement de compétences, méthodes et procédures. A l'intérieur de cette réalité, la littérature risque de perdre sa valeur initiale et la transmission se fait de plus en plus en partant d'un questionnement méthodologique duquel surgit un nouveau cadre programmatique où le plus important est, à notre avis, l'interaction entre théorie et praxis (Bárcena, 2005). Le passé doit être pris en compte dans la mesure où il éclaire le champ de l'actuel qui se débat entre le présent et le passé. Or, cette imbrication exige certaines considérations. $\mathrm{Si}$, en effet, la lecture se situe au cœur d'une nouvelle méthodologie, l'incorporation d'un corpus plus varié de textes peut contribuer à déplacer l'étude de la littérature car, au plan méthodologique, elle peut se voir absorbée par l'enseignement de la lecture, et en particulier par tous les dispositifs techniques de la lecture littéraire. Cette conception vide le sens global des œuvres littéraires des éléments supra textuels. A ce propos Jean-Louis Dufays observe que :

Dans les différents pays francophones, l'enseignement de la littérature (...) est depuis une trentaine d'années le lieu d'une tension entre deux grands paradigmes, généralement perçus comme antagonistes, l'un qui privilégie la connaissance du patrimoine littéraire, l'autre qui privilégie la pratique méthodique de la lecture (Dufays, $2006: 7)$. 
En même temps, cette nouvelle réalité, qui est également valable au niveau de l'enseignement supérieur, suppose, au cadre théorique, une double possibilité qui enrichit la dimension heuristique de la littérature. C'est justement la praxis (action) qui a besoin d'une coopération littéraire sur laquelle se construit une poiésis (production), qui offre la possibilité à celui qui apprend d'aller, au moyen de la littérature, à la découverte du sens, de soi et de l'altérité. Les deux tendances (soit, le maintient du patrimoine, d'un côté, et soit la lecture critique, de l'autre) ne doivent pas se présenter comme irréconciliables au plan de la transmission des contenus et de l'acquisition des compétences. Notons en outre, qu'une typologie rigoureuse a été déjà établie, et, en matière de l'explication de textes littéraires, elle ouvre la réflexion du modèle pédagogique vers la pédagogie du projet faisant de l'apprenant un acteur social : "la pédagogie du projet se révèle un dispositif permettant de varier les relations tâches-actions et de donner un sens à toutes les tâches (...) en les mettant constamment en relation directe avec des actions sociales " (Dufays, 2006 : 10). Les données de cette typologie suivent le classement inductif proposé par le CECR (Cadre Européen Commun de Référence) de 2001 et donnent comme résultat l'analyse actionnelle axée sur des compétences, des attitudes et des valeurs qui permettront à l'étudiant par la suite une meilleure intégration professionnelle: «Si les actes de parole se réalisent dans des activités langagières, celles-ci s'inscrivent elles-mêmes à l'intérieur d'actions en contexte social qui seules leur donnent leur pleine signification » (Consejo de Europa, CECR, 2001 : 14-19) $)^{4}$.

Au sens large, l'enseignement de la littérature au niveau supérieur peut être conçu, à notre avis, comme un ensemble complexe articulé autour de sphères d'interférences diverses où l'étudiant apprend à se situer, soit au plan conceptuel, soit au plan figuratif, au moyen du raisonnement analogique et notamment à ressentir la littérature comme une expérience esthétique, voire une expérience intime. La littérature peut donc être réécrite et donc devenir un véritable «faire social » valable pour la formation de «l'acteur social » qu'est l'apprenant, à fortiori l'étudiant universitaire.

\footnotetext{
${ }^{4}$ La typologie obéit à un classement inductif des consignes suivantes: Paraphraser, Analyser, Interpréter, Extrapoler, Comparer, Réagir et Transposer.
} 


\title{
L'enseignement de la littérature interculturelle francophone
}

\begin{abstract}
Mais pourquoi s'arrêter à la littérature française ? (...) II est temps, me semble-t-il, de faire éclater les frontières linguistiques puisque l'héritage national ne joue plus un rôle essentiel, et une même crise se manifeste un peu partout.
\end{abstract}

Alter, $1981: 62$

En tenant compte de la double perspective conceptuelle et méthodologique, nous avons proposé depuis l'année dernière, dans le cadre du programme des études officielles de Master de l'Université Autonome de Madrid, l'approche interculturelle en rapport avec le caractère plurilingue de l'Europe actuelle qui travaille en faveur du rapprochement et du dialogue. De ce point de vue, l'intercompréhension s'instaure de plus en plus comme une voie d'enrichissement et d'ouverture (Rencontres, 2006) ${ }^{5}$. Ce Master, intitulé «Le Français dans le cadre professionnel: des connaissances théoriques aux compétences professionnelles», propose essentiellement un parcours professionnel (60 crédits), mais n'exclut pas la formation dans le domaine de la recherche (60 crédits). Les deux orientations sont articulées autour de plusieurs modules axés sur une formation compréhensive et réflexive en matière de langue, communication, traduction, civilisation et littérature. L'accent est mis sur la formation pluridimensionnelle ainsi que sur la satisfaction des étudiants qui, en général, ce sont des professeurs consacrés à l'enseignement secondaire. En plus, il s'agit d'un Master officiel accepté par la Communauté Autonome de Madrid où la langue et la culture françaises jouent un rôle fondamental et dont la qualité éducative est reliée aux équipements, aux nouvelles technologies, aux ressources et aux profils professionnels.

C'est dans ce sens que nous travaillons dans le cursus intitulé Littératures des pays francophones à la frontière entre le pluralisme axiologique et la cohérence culturelle. En effet, nous avons conçu l'étude de la littérature française contemporaine et des littératures francophones dans un contexte plus large, du point de vue culturel, intellectuel, institutionnel, historique et social. L'objectif axial a été donc d'intégrer les connaissances encyclopédiques, d'une part, l'interculturalité et le plurilinguisme, de l'autre. Le programme est articulé en partant de la polarité littérature nationale française / littérature interculturelle francophone afin de montrer les points de contact où se profilent des charnières permettant des médiations.

\footnotetext{
${ }^{5}$ À l'occasion du salon Expolangues (Porte de Versailles du 17 au 21 janvier 2006), la délégation générale à la langue française et aux langues de France a organisé une table ronde sur l'intercompréhension entre langues apparentées.
} 
Plus particulièrement nous travaillons le champ de la littérature ectopique ${ }^{6}$ (Albaladejo, 2007), déterritorialisée, par opposition à la notion de littérature nationale enracinée historiquement. Ce type de littérature comporte des caractéristiques singulières dont les plus significatives sont : le changement de territoire (associé notamment à des situations d'exil, immigration, voyage-projet), la rencontre avec l'altérité, l'emploi d'une langue différente de celle de la langue première, la dénonciation idéologique et la réflexion autour de l'écriture artistique et de ses possibles manifestations formelles et thématiques comme voie d'un nouveau projet de vie intégré. Le plus important, de ce point de vue, est l'étude de la nouvelle poétique qui dérive de l'expérience de brassage et d'hybridation. Une transpoétique qui tisse une nouvelle sensibilité sans ignorer les référents du passé.

A ce propos, Hédi Bouraoui anime consciemment le néologisme créaculture pour «(mettre) l'accent sur le côté créateur des valeurs représentant une vision unitaire où le sujet et le monde sont en perpétuelles tensions et créations vers une éventuelle harmonie » (Bouaroui, 2005 : 32). Pour cet auteur il y a quatre composantes de la créaculture, «le préfixe créa du mot création ajouté à la culture représente la transformation métaphysique de la linéarité évolutive idéelle, sensible, visible et matérialiste, mettant l'accent sur le dynamisme d'une culture qui se fait » :

1. Elle étudie les structures fondatrices d'une civilisation pour saisir les modalités, dégager les notions clés, les constantes qui informent l'unité sous-jacente.

2. Elle met en lumière le dynamisme de la culture en perpétuel devenir, l'évolution et la métamorphose de ces dominances culturelles.

3. Elle centre le débat sur le présent ; le passé est pris en compte dans la mesure où il éclaire le champ de l'actuel.

4. Elle souligne particulièrement le côté créateur de ces idées-forces à partir des chocs culturels selon l'analyse constrastive (Bouaroui, 2005 : 33).

Par ailleurs, nous choisissons l'approche contextuelle qui nous permet de mettre en valeur les interactions des différents champs littéraires et d'étudier un corpus varié d'écrivains qui thématisent l'hybridation et enrichissent le canon esthétique traditionnel à la frontière d'une littérature nationale ${ }^{7}$. Le phénomène littéraire peut donc être abordé en tenant compte des références culturelles où certains auteurs marquent les ruptures et les symbioses du fait littéraire et contribuent à l'évolution au-delà des canons acceptés. De

\footnotetext{
6 L'expression "littérature ectopique" (du grec ektopos, indique l'éloignement de sa place) recouvre les phénomènes de la déterritorialisation, reterritorialisation et transterritorialisation auxquels participent un nombre important d'écrivains représentatifs de la littérature interculturelle contemporaine.

7 Cf notre travail à propos de l'œuvre littéraire d'Agota Kristof (1936-), auteure d'origine hongroise, exilée en Suisse depuis 1956. La trilogie des jumeaux illustre en langue française (langue d'adoption) la richesse de son écriture à la frontière de deux traditions littéraires, la hongroise et la française (Alfaro, 2007, 2008).
} 
même, la littérature interculturelle promeut la connectivité sociale, d'après l'expression d'Edgard Morin. Le dialogue interculturel permet l'établissement d'une pédagogie interculturelle qui peut éviter les attitudes autoritaires et rigides de l'enseignement. Bien entendu, le travail de l'enseignant devient notamment le lieu d'un transfert où connaissance théorique, interprétation analytique et participation ouvrent la voie vers l'intercompréhension.

\section{Conclusion}

En somme, l'enseignement de la littérature et ses réseaux littéraires peut se transformer dans les nouveaux plans d'études de l'enseignement supérieur en un «lieu d'une tension entre deux grands paradigmes, généralement perçus comme antagonistes, l'un qui privilégie la connaissance du patrimoine littéraire, l'autre qui privilégie la pratique méthodique de la lecture»

Cependant, certains défis surgissent de la réalité sociale et économique. Depuis quelques décennies, la quête d'une citoyenneté stable oblige les états membres de l'Union à incorporer sans cesse des lois et des contextes légaux dans leurs réalités nationales, visant un progrès socio-économique optimal. Or, au sein de la pensée intellectuelle, la réflexion a été menée essentiellement d'un point de vue sociologique en raison des intenses mouvements migratoires que les démocraties occidentales connaissent. Au-delà de la constatation de ces circonstances, dans le domaine des Humanités (la transmission des savoirs) le projet éducatif doit évoluer vers une expérience compréhensive mais notamment vers une expérience réflexive. Enseigner la matière de littérature L.E. devrait contempler l'établissement d'un plan de formation intégral et continue entre l'enseignement primaire, secondaire et universitaire ainsi que la formation de futurs enseignants. En plus, dès la perspective méthodologique et didactique, la littérature actuellement semble, au cadre institutionnel de l'enseignement supérieur, devoir s'adapter plutôt à l'approche par compétences et capacités (savoir faire) au détriment de l'apprentissage de savoirs conceptuels (historiques, techniques et génériques).

Nous constatons que le plus important est, en dehors des bipartitions et des dichotomies, que la littérature est «un domaine disciplinaire complexe et multiforme» qui offre à l'étudiant la possibilité de s'ouvrir à de nouvelles attitudes (savoir être) telles qu'apprendre à vivre, agir et penser en situation d'incertitude et face à une diversité d'approches. Nos étudiants « aurons reçu (de l'œuvre littéraire) les règles d'une démarche qui leur servira dans l'expérience du vécu » (Alter, 1981:61-63).

Pour conclure, une question sous-jacente nous interpelle. Question qui se trouve, sans doute, à la base de nos discussions intrauniversitaires que les représentants des sciences expérimentales et des programmes techniques nous posent aux professeurs de 
littérature des langues étrangères que nous sommes. La question est la suivante : Pourquoi, donc, enseigner la littérature? La réponse donnée en 1969, lors du colloque de Cerisy organisé par Serge Doubrovsky et Tzvetan Todorov- et auquel ont participé des critiques aussi célèbres que Genette, Rifaterre, Greimas, Barthes, Pingaud, Alter, était la suivante :

.... parce que nous vivons dans un monde inquiet, dans une société bouleversée, dans une culture en transition, où les réponses traditionnelles se vident de substance, où des nouvelles questions se posent, où les sciences pures, les sciences appliquées et, surtout, les sciences sociales montent à l'assaut des humanités (...) (Alter, 1981 : $58)$.

Quatre décennies plus tard, l'enjeu majeur de l'enseignement supérieur est l'innovation, et l'enjeu de l'enseignement de la littérature aujourd'hui est de viser, certes, la complexité de la mondialisation par le biais de la communication interculturelle et, en plus, de repenser la notion de patrimoine littéraire ayant comme horizon l'identité littéraire de l'Europe (Steiner, 2007 ; Fumaroli et al., 2000). D'après notre expérience, l'étude des littératures francophones à la lumière de la littérature française, depuis la perspective interculturelle, "nous invite à rompre avec cette approche exclusivement nationale, à dissocier littérature et langue nationale et à reconnaître la dimension pluriculturelle de la langue française » (Houdart-Merot, $2006:$ 85).

Pour ce faire, une mission s'impose aux professeurs de littérature au profit $d u$ dialogue interculturel. Nous citons Edgard Morin qui dresse la hiérarchie de cette noble mission éducatrice:

- proporcionar una cultura que permita distinguir, contextualizar, globalizar, enfrentarse con los problemas multidimensionales, globales y fundamentales ;

- preparar los espíritus para hacer frente a las incertidumbres que no cesan de crecer, no sólo haciéndoles descubrir la historia insegura y aleatoria del Universo de la vida, de la humanidad, sino favoreciendo en ellos la inteligencia estratégica y la apuesta por un mundo mejor ;

- educar para la comprensión humana ente próximos y lejanos ;

- enseñar la afiliación a Francia, a su historia, a su cultura, a la ciudadanía republicana, e introducir la afiliación a Europa (Morin, 2000: 133). 


\section{Bibliographie}

AguAdO, Teresa (2003). Pedagogía intercultural. Madrid : MacGraw Hill

AlbalADEJO, Tomás (2007). «Ectopic Literature». In : Papeles de trabajo del Grupo de Investigación C [PyR]. Madrid, UAM.

ALFARO, Margarita (1998). "Los procesos de la actividad docente ». In : HOLZBACHER, Ana Mㄹ, SUÁREZ, Pilar (éds) (1998). Reflexiones sobre la enseñanza de la literatura. Madrid, Ediciones de la UAM, pp. 77-80.

AlfaRo, Margarita (2007). " Escribir en la frontera. Exilio y escritura en la trilogía de Agota Kristof ». In : ALFARO, Margarita et al, Más allá de la frontera : cinco voces para Europa. Madrid, Calambur, pp. 19-51.

ALFARO, Margarita (2008). « Exil et création littéraire au sein de l'Europe contemporaine ». In : Latitudes. Espaces transnationaux et imaginaires nomades en Europe. CRTF, Cergy-Pontoise, pp. 125-136.

ALTER, Jean (1981). « Pourquoi enseigner la littérature ?. In : DOUBROVSKY, Serge, TODOROV, Tzvetan (orgs). L'enseignement de la littérature. Paris, Duculot, pp 53-63.

BÁRCENA, Fernando (2005). La experiencia reflexiva en educación. Madrid, Paidós.

BouRAOUI, Hédi (2005). Transpoétique. Éloge du nomadisme. Montréal, Mémoire d'encrier.

CONSEJO DE EUROPA (2001) European Languaje Portafolio del Consejo de Europa. Modern Languajes. Versión española : Marco de referencia europeo, la enseñanza y la evaluación de lenguas. $<U R L$ : http://cvc.cervantes.es/obref/marco/>.

CuCHE, Denys (2004). La notion de culture dans les sciences sociales. Paris, La Découverte.

DOUBROVSKY, Serge, TODOROV, Tzvetan (orgs) (1981). L'enseignement de la littérature. Paris, Duculot.

DOUBROVSKY, Serge (1981). «Le point de vue du professeur ». In : DouBROVSKY, Serge, TODOROV, Tzvetan (orgs). L'enseignement de la littérature. Paris, Duculot, pp. 7-19.

DUFAYS, Jean-Louis (2006). "La lecture littéraire, des « pratiques du terrain » aux modèles théoriques ». In : LIDIL (Revue de Lingusitique et de didactique des langues), 33, pp. 79-101. $<U R L$ : http://lidil.revues.org/document60.html>

FERNÁNDEZ, Ma del Carmen (2008). « Aproximación a la historia del concepto de canon en Francia y a su configuración actual ». In : Espéculo. Revista de Estudios Literarios. UCM. <URL : http://www.ucm.es/info/especulo/numero 38/canonfr.html>.

FUMAROLI, Marc et al. Identité littéraire de l'Europe. Paris, PUF.

GOLDENSTEIN, Jean-Pierre (1998). « Savoir-lire littéraires et enseignement universitaire actuel de la littérature ». In : HOLZBACHER, Ana Mํㅡ, SUÁREZ, Pilar (éds). Reflexiones sobre la enseñanza de la literatura. Madrid, Ediciones de la UAM, pp. 35-49.

HOLZBACHER, Ana Mạ SUÁREZ, Pilar (éds) (1998). Reflexiones sobre la enseñanza de la literatura. Madrid, Ediciones de la UAM.

HOUDART-MEROT, Violaine (2006). « Convergences entre littératures francophones et littérature française : les écritures babéliennes ». In : Convergences Francophones. Cergy-Pontoise, CRTF, pp 69-85

MONTAIGNE, Michel de (1969). Essais, Livre I. Paris, Garnier Flammarion.

MORIN, Edgar (2000). La mente bien ordenada. Barcelona, Seix Barral.

PUREN, Christian (2006). «Explication de textes et perspective actionnelle : la littérature entre le dire scolaire et le faire social ». In : CELEC-CEDICLEC. <www.dlc.sup.fr>

STEINER, George (2007). La idea de Europa. Madrid, Siruela.

TODOROV, Tzvetan (2005). La peur des barbares. Paris, Robert Laffont. 
UNESCO-CEPES (2005). L'Enseignement supérieur en Europe. Dialogues de développement dans le contexte de l'éducation pour tous et de l'assurance de la qualité dans l'enseignement supérieur. Vol XXX, No. 3-4. 